\title{
24 A critical reflection on Photovoice applied in exploring the lived experience and needs of male caregivers
}

\author{
Frances Lu Yang, Vivian Wei Qun Lou \\ and Carman Ka-Man To
}

\begin{abstract}
Photovoice allows participants to speak freely about their life and roles by using the camera to capture their first-hand situations in photographs, and by later recalling the related stories. On the one hand, this methodology shifts the focus to involving participants in order to amplify their own voices and thus enhance their agency; but, on the other hand, for certain groups of people - in particular, the most deprived populations who have unequal power relations with scholars - this authorization still falls under the researcher's dominance in the details of the operation. Therefore this chapter argues Photovoice should focus on empowering participants to establish stronger social networks and to improve their local communities, and it should always be operated with innovative measures that consider contextual influence.
\end{abstract}

\section{Introduction}

Unlike traditional methodology, visual materials are no longer used exclusively by researchers. Photovoice is a participatory method that allows participants to speak freely about their life and roles, as they use the camera to capture their first-hand situations in photographs and later recall the related stories (Angelo and Egan, 2015). Voice involves "voicing our individual and collective experience" (Wang and Burris, 1997, p. 381). Participatory methods of gaining new information involve participants addressing issues that are important to them; in this context, researchers are able to create knowledge that is more closely centered on the experiences of individuals, rather than viewing people simply as data sources and typically subjects of research trials (Letts, 2003).

Rooted in the three theoretical assumptions of critical consciousness, feminist theory and documentary photography (Wang and Burris, 1997), Photovoice creates spaces and opportunities for marginalized voices to be heard. Grounded in lived experience, Photovoice can help communities identify, discuss and resolve significant structural problems as they seek to use social action to inform policy. Having participants create knowledge based on their lived experiences can lead to the development new ways of seeing and understanding the context of social problems; participant-created data has the potential to challenge stereotypes and offer alternative explanations of the causes of, and answers to, social problems. In terms of narrative analysis, Photovoice offers first-hand experiences and inspires researchers to explore from more perspectives (Angelo and Egan, 2015). It allows investigators to gain an intimate familiarity with the community in which participants live and make participants' voices heard by stakeholders and policymakers. 
Photovoice has been gaining popularity as a participatory research methodology and has been applied widely in studying populations from various socio-cultural backgrounds, including gender minorities (Harcourt, 2013), youth (Ardiles et al., 2019), the elderly (Novek, Morris-Oswald and Menec, 2012), homeless people (Seitz and Strack, 2016) and people with intellectual disabilities (Povee, Bishop and Roberts, 2014). This qualitative method of inquiry is mainly used for three purposes: (1) to provide a forum to present participants' lived experiences through the images, language and contexts defined by participants themselves and, based on that, to identify the strengths and weaknesses of their community; (2) to promote critical dialog and knowledge about personal and community issues; and (3) to approach policymakers in the participants' area (Wang, 2006).

\section{Contextualize Photovoice in male caregiver study}

The project Defining Sonhood: The Exploration of Lived Experience of Caregiving Sons in their Late Adulthood in Hong Kong is an exploratory study in which researchers attempt to conceptualize a new framework on solid field studies into community and to conduct a deep investigation into caregiving sons' lived experiences. Photovoice was selected as the dominant methodology and was supplemented by interviews and focus groups. As a participatory method, Photovoice fits the criterion of allowing caregivers to speak freely about their role. To thoroughly understand the lives and needs of this group, the research project has three major aims. The first aim is to examine factors that predispose sons to take on a caregiving role and to see how sons manage this role in their later adult life. The second aim is to investigate the lived experiences of caregiving sons in Hong Kong, mainly from three aspects: how they define caregiving, pains and gains in caring for their parents, how the caregiving role impacts other aspects of their individual life and to explore the practical implications of conceptualizing sonhood for social issues.

Photovoice was used as a primary method to collect the data, whereas interviews were used to prompt participants to explain their stories, mainly including experiences associated with the photos, notes and photo-taking process. The photos encourage participants to reflect on their experiences through their own lens. In addition, interviews were used to investigate the factors of being caregiving sons, their pains and gains in caring for their parents and how their caregiving role impacts other aspects of their individual life.

Research on caregiver burden is predominantly quantitative in nature (Adelman et al., 2014). It associates lack of support for daily caregiving tasks with higher caregiver burden (Kaufman et al., 2010) and poor caregiver quality of life with physical and mental health issues (Chang, Chiou and Chen, 2010). Quantitative research concentrates on the categories of specific caregiving tasks, the level of the burden and the influence of the burden on caregivers. However, no official statistics focus on male family caregivers in Hong Kong, and, in academia, no solid and systematic studies have been conducted to understand how men perceive their roles and caregiving behaviors when age and family relationships intrude in different combinations.

As a result, Photovoice was chosen as an appropriate method to capture the reality of people's lives and to present these realities and moments to others. Photovoice thus provides an opportunity for male caregivers to present their lived experiences through images and language. Physical environments and narrations of the photos defined the contexts in which the strengths and weaknesses of the local community were examined by the participants themselves. 
As reviewed in the above section, Photovoice is widely accepted in studying minorities and marginalized populations. Men, a group not traditionally recognized as caregivers, are rising to the occasion. Conventionally, women have been assumed as nurturers and the primary caregivers in the family, including in Hong Kong (Cheung and Halpern, 2010) while men have been established as breadwinners who focus on the external world. As a gendered concept, caregiving is often defined as feminine, and caregivers are treated as a subordinate class; caring practice engenders a feminine identity, while masculinity is seen as incompatible with caring skills (Hanlon, 2009). In that case, male caregivers were socially constructed as a marginalized group.

As described above, Photovoice was supplemented by message descriptions and interviews to examine this marginalized group. Caregiving was regarded as a practice-based framework defined by narration of the photos, which record the caregiving experiences of specific moments.

The targeted sample is caregiving sons in later adulthood (aged 45-65) in Hong Kong, and 60 participants are invited to participate. Due to the fact that Hong Kong does not have official statistics on caregiving sons, it is impossible to employ random sampling. Furthermore, scholars have pointed out that hegemonic masculinity makes men too anxious to accept the caregiver role (Hearn, 2004): the conflict between the social expectations of men and the emotional abilities required for caregiving leads to rejection of a caregiver identity. In addition, it is not common for men to seek help and emotional support from the community.

As such, besides purposive sampling through non-government organizations (NGOs) and the researchers' personal networks, peer research was also employed for recruitment. Purposeful sampling was adopted, in which specific socio-demographic characteristics of Hong Kong caregiving sons will be considered in setting up the inclusion and exclusion criteria for sample recruitment. Peer research is applied to studies involving hard-to-reach populations (O’Reilly-de Brún et al., 2015). By borrowing the praxis of peer research for recruitment, this study could reach greater numbers of a marginalized group, with the collected data more accurately representing community needs and increasing opportunities for recognition of self-identity and empowerment.

\section{The modification of Photovoice}

The whole process of Photovoice includes four major parts: (1) participants are required to take photos portraying their experience of the investigated phenomenon; (2) participants illustrate what they want to express through the photos; (3) researchers contextualize the photos by analyzing them and their narrations; (4) interviews and focus groups elicit the story behind the photos in order to further understand participants' caregiving experiences. These four parts are attained through a standardized procedure with four meeting sessions, including the orientation session, the photo collection with elicitation interview session, the reflection session and the group discussion session.

This project modified the above procedure. The data is collected more flexibly, with photo collection and interviews. The whole procedure includes four major steps: (1) proposed protocol based on literature review; (2) capacity building of researchers; (3) refined protocol; (4) coping with the challenges encountered during initial recruitment and adjusting protocol. During the initial recruitment in February and March 2019, ten successfully contacted male caregivers as well as partnered NGOs disclosed their difficulties in committing to attending four sessions of Photovoice, since they usually have full-time jobs and/or 
other engagements in their personal lives. It is very hard for them to spare time for four sessions in busy, fast-paced Hong Kong. Although they are interested in participating in this project, four session-designed activities are too demanding for them. Moreover, participants expressed their concern about disclosing about their personal lives in front of strangers.

Meanwhile, as mobile devices become advanced and popular among the general population, the technology literacy of using smart phone apps - such as mobile cameras - is increasing among older adults. Most participants have already acquired skills to use multifunction instant messaging apps for communication (e.g., WhatsApp), and most participants were familiar with taking photos by smart phone. As a result, the methodological operation was redesigned, emphasizing flexibility throughout the whole process. The sessions required by the traditional Photovoice flow can be conducted in an innovative way. For instance, interviews and discussions can be conducted via phone, email or instant messenger apps such as WhatsApp, while photos can be collected through tools such as Google Drive and email.

\section{Discussion}

This participatory approach aims to promote critical dialog and knowledge about personal and community issues. Unlike other methods that do not penetrate the community, Photovoice enables participants to work as partners and collaborators of the researchers. In a social process of critical consciousness (Carlson, Engebretson and Chamberlain, 2006), researchers help individuals express their own experiences by critically reflecting their assumptions and interpretations of reality. Participants are only able to conduct dialog with relevant policymakers based on their understanding of their identity as caregiver and the situation they were experiencing (Wang, 2006).

To realize this goal by research-participant collaboration, two critical issues should be faced throughout the process: (1) the balance between the role of researchers' decisionmaking in the process on the one hand, and participants' dominance in portraying their experiences by photography on the other hand; and (2) the barriers of and empowerment from using the Photovoice technique among participants. Meanwhile, methodological strengths and weaknesses were reflected on to improve the Photovoice application throughout the entire project.

\section{The balance between the role of researchers' decision-making in the process on the one hand, and participants' dominance in portraying their experiences by photography on the other hand}

The utilization of Photovoice was based on the balance between researchers' decisionmaking and participants' dominance in portraying their experiences. In Photovoice studies, the relationship of researchers and participants cannot simply be described in terms of the outsider-insider (Fletcher and Kerr, 2010) and dominance-obedience binaries. Data collection is completed by the participants, which engages and enables community members to participate as partners/collaborators in the research process (Porter, 2016). From three dimensions, researchers and participants worked as partners and collaborators throughout the process of this by balancing the dynamic between the researcher's decision-making and the participants' articulation. 
First, researchers and participants co-defined the term "male caregiver" and coconceptualized the framework of "caregiving". As stated above, the conceptualization of "caregiving and caregiver" has been feminized; consequently, the description of the inclusion criteria was modified after interactions between researchers and participants. Before the recruitment, researchers defined "primary caregiver" as someone who provided no less than four hours of care per week to one or both parents, or who visited parents who live in nursing homes and providing assistance in care on a regular basis.

With the first round of recruitment, it was found that potential candidates had different understandings of "caregiving" and "primary caregiver", which was not expected when recruiting female counterparts. A woman could definitely identify herself as a primary caregiver by counting caregiving hours and providing a straightforward framework of caregiving tasks; however, a man could hardly articulate his caregiving contribution to this framework. One participant's response was representative of the sample:

I am confused about the definition of "caregiver". I think I would recognize myself as a primary caregiver if I stayed up all night to guide the domestic helper and I experienced pressure as both a caregiver and a son. But I did not physically assist in showering or toilet use by myself if you define those tasks as caregiving.

For this reason, the definition of the targeted population in the recruitment was modified by adding "self-identity as a primary caregiver" and excluding those who only provide financial assistance.

Second, researchers and participants co-create the methodological procedure. At the preparation stage, researchers designed the study to strictly follow the theoretical flow, as detailed above. However, in reality, not all the participants in the targeted age group ("later adulthood") were retired, and, as such, some participants still had a full-time job besides their intensive caregiving workload. Even if professionals - including social workers have taken the lead in the research, placing additional burdens on this group for research purposes cannot be justified.

As a result, the operating flow of Photovoice has been adjusted based on the convenience of the participants and the theoretical coherence of the methodology. All the photos were collected via WhatsApp as soon as they were taken; instead of conducting photoelicitation interviews and focus groups in the final session, researchers conducted interviews and focus groups on a more flexible schedule. Participants were invited to take part in phone or face-to-face interviews if they were unable to attend the session. Furthermore, to guarantee minimal influence on outcomes, participants were asked to write short instant memos to describe the photos when sending them to the researchers.

Third, the researchers and the participants co-interpret the photos. Photovoice gives the participants voice and enhances their agency to express their stories, but, at the same time, it also requires researchers to make decisions in order to attain the research aims. In this project, participants took photos following by a set of guidelines:

- take photos to show what your current life is like;

- $\quad$ take photos to convey your concerns when caring for your parents;

- take photos to convey what you think relates to caregiving;

- take photos to show your greatest contribution to taking care of your parents;

- take photos to most accurately represent the relationship with the one you give care to;

- take photos to show your individual life outside taking care of your parents. 
This process was carried out from an "insider" perspective; participants were encouraged to define and depict "caregiving" according to their own understanding. The SHOWeD approach (Gant et al., 2009) was applied to interpret the photos from an "outsider" perspective, in order to examine the related social issues and to facilitate policymaking. The question guideline is listed below.

\section{SHOWeD Approach (Gant et al., 2009)}

- What do we see in this picture?

- What are we seeing in this picture?

- What is this picture about?

- How does this picture relate to your life here?

- What should we/other people understand about your experience from this picture?

- What do you want others who see this picture to understand about your experience as shown in the picture?

- What are your thoughts/feelings about this picture?

- Why do these issues exist?

- How can we become empowered by our new social understandings?

- What can we do to address these issues?

- Given what we have learned so far, what should we explore next?

By probing into the questions above, participants and researchers collaborate to give thick descriptions of relevant social issues, which give rise to increased understandings of contextual influence.

\section{The barriers of and empowerment from using Photovoice among participants}

As stated above, Photovoice can empower participants; it authorizes their voice to articulate caregiving experience by taking photos and reflectively narrating those photos. Photographs capturing physical and social environments drew out a range of emotions and experiences in different settings. However, some participants were resistant to learning how to use smart phones and social media, a phenomenon that is more common among the targeted population of this study. Other challenges also repeatedly occurred during the study, such as participants preferring words and conversations over taking photos to present their sentimental issues, having concerns about sharing personal photos with others and finding Photovoice to be time consuming and just another burden to their workload.

At the same time, the method of Photovoice to some degree marginalized institutional ideologies/arrangements and facilitated more critical thinking of related social issues. Practically, in those caregivers' personal lives, they better understood their roles as caregivers and developed critical awareness of their community; this reflection made them feel more at ease in taking on the caregiving role. Participants developed close attachments and better relationships with their parents by recalling memories and collecting photos. Lastly, this study allowed participants to expand their social networks among peers and to build connections with professionals (research partners and policymakers). 


\section{Reflection on methodological strengths and weaknesses of practical Photovoice use}

As seen in the above analysis, practical issues and challenges in methodological practice sometimes conflicted with the theoretical principle of Photovoice as "liberatory enactment" (Malherbe et al., 2017, p. 165). As a participatory, collaborative visual study, Photovoice is "a process by which people can identify, represent, and enhance their community through specific photographic technique" (Wang and Burris, 1997, p. 369). Scholars proposed that, unlike focusing on researchers' dominance in traditional praxis, Photovoice shifts the focus to involving participants in order to amplify their own voices and thus enhance their agency.

However, for certain groups of people - in particular, the most deprived populations who have unequal power relations with scholars - this authorization still falls under the researcher's dominance in the details of the operation. In this project, participants of older ages and with lower education levels tended to quit during the process. In other words, rather than emphasizing a diminishing of the imbalance between the participants and the researchers, Photovoice should focus on empowering participants to establish stronger social networks and to improve their local communities. To that end, Photovoice should always be operated with innovative measures that consider contextual influence.

\section{Conclusion}

To conclude, this chapter briefly explained how Photovoice has been conceptualized and how it is increasingly being used in social science research, particularly in studying marginalized populations in the areas of public health and social work. To be specific, this study used a research project on sons as caregivers in Hong Kong to illustrate how Photovoice was flexibly used, along with the theoretical assumptions of the modification.

The fundamental aim of Photovoice was to empower the participants' voices by providing a forum for participants to present their lived experiences through images, language and contexts defined by participants themselves, thus enabling participants to promote dialog and knowledge about their own identities and the strengths and weaknesses of the local community. The innovative way to collect the data reminded the researchers to balance the role of their decision-making with participants' dominance in portraying and interpreting their experiences throughout the process. In addition, the barriers of and the empowerment from using IT-supported techniques and social media were also reflected on. It was necessary to understand how the technique could shift the power dynamic between the researcher and the marginalized population, at the same time keeping in mind the potential barriers and possibilities of changing of power relations.

Finally, the ultimate goal of using Photovoice is to establish a stronger community by informing the broader, powerful society. Photos and the stories accompanying them are encouraged to be exhibited to the public with ethical permission, which would help create a space for conversation between participants and their local community.

\section{Acknowledgement}

This research project (Project Number: 2017.A8.076.18B) is funded by the Public Policy Research Funding Scheme from the Policy Innovation and Co-ordination Office of The Government of the Hong Kong Special Administrative Region.

\section{References}

Adelman, R.D., Tmanova, L.L., Delgado, D., Dion, S. and Lachs, M.S. (2014). Caregiver burden: a clinical review. JAMA, 311(10), 1052-1060. 
Angelo, J. and Egan, R. (2015). Family caregivers voice their needs: a Photovoice study. Palliative and Supportive Care, 13(3), 701-712.

Ardiles, P., Casteleijn, M., Black, C. and Sørensen, K. (2019). Using Photovoice as a participatory approach to promote youth health literacy. In: O. Okan, U. Bauer, D. Levin-Zamir, P. Pinheiro and Sørensen, K. (eds), International handbook of health literacy: research, practice and policy across the life-span. Bristol, UK: Policy Press.

Carlson, E.D., Engebretson, J. and Chamberlain, R.M. (2006). Photovoice as a social process of critical consciousness. Qualitative Health Research, 16(6), 836-852.

Chang, H.Y., Chiou, C.J. and Chen, N.S. (2010). Impact of mental health and caregiver burden on family caregivers' physical health. Archives of Gerontology and Geriatrics, 50(3), 267-271.

Cheung, F.M. and Halpern, D.F. (2010). Women at the top: powerful leaders define success as work + family in a culture of gender. American Psychologist, 65(3), 182.

Fletcher, G.J. and Kerr, P.S. (2010). Through the eyes of love: reality and illusion in intimate relationships. Psychological Bulletin, 136(4), 627.

Gant, L.M., Shimshock, K., Allen-Meares, P., Smith, L., Miller, P., Hollingsworth, L. A. and Shanks, T. (2009). Effects of Photovoice: civic engagement among older youth in urban communities. Journal of Community Practice, 17(4), 358-376.

Hanlon, N. (2009). Caregiving masculinities: an exploratory analysis. In: K. Lynch, J. Baker and M. Lyons (eds), Affective equality: love, care and injustice. Basingstoke, UK: Palgrave Macmillan.

Harcourt, W. (2013). Body politics in development: Critical debates in gender and development. London: Zed Books Ltd

Hearn, J. (2004). From hegemonic masculinity to the hegemony of men. Feminist Theory, 5(1), 49-72.

Kaufman, A.V., Kosberg, J.I., Leeper, J.D. and Tang, M. (2010). Social support, caregiver burden, and life satisfaction in a sample of rural African American and White caregivers of older persons with dementia. Journal of Gerontological Social Work, 53(3), 251-269.

Letts, L. (2003). Occupational therapy and participatory research: a partnership worth pursuing. American Journal of Occupational Therapy, 57(1), 77-87.

Malherbe, N., Suffla, S., Seedat, M. and Bawa, U. (2017). Photovoice as liberatory enactment: the case of youth as epistemic agents. In: M. Seedat, S. Suffla and D.J. Christie (eds), Emancipatory and participatory methodologies in peace, critical, and community psychology. Cham: Springer.

Novek, S., Morris-Oswald, T. and Menec, V. (2012). Using Photovoice with older adults: some methodological strengths and issues. Ageing \& Society, 32(3), 451-470.

O’Reilly-de Brún, M., de Brún, T., Okonkwo, E., Bonsenge-Bokanga, J.S., Silva, M.M.D.A., Ogbebor, F., Mierzejewska, A., Nnadi, L., van Weel-Baumgarten, E., van Weel, C., van den Muijsenbergh, M. and MacFarlane, A. (2015). Using participatory learning and action research to access and engage with "hard to reach" migrants in primary healthcare research. BMC Health Services Research, 16(1), 25.

Porter, G. (2016). Reflections on co-investigation through peer research with young people and older people in sub-Saharan Africa. Qualitative Research, 16(3), 293-304.

Povee, K., Bishop, B.J. and Roberts, L.D. (2014). The use of Photovoice with people with intellectual disabilities: reflections, challenges and opportunities. Disability \& Society, 29(6), 893-907.

Salifu, Y., Almack, K. and Caswell, G. (2018). The changes, challenges, and coping strategies of men living with advanced prostate cancer in a resource-poor country. Journal of Pain and Symptom Management, 56(6), e71-e71.

Seitz, C.M. and Strack, R.W. (2016). Conducting public health Photovoice projects with those who are homeless: a review of the literature. Journal of Social Distress and the Homeless, 25(1), $33-40$.

Wang, C.C. (2006). Youth participation in Photovoice as a strategy for community change. Journal of Community Practice, 14(1-2), 147-161.

Wang, C. and Burris, M.A. (1997). Photovoice: concept, methodology, and use for participatory needs assessment. Health Education and Behaviour, 24, 369-387. 